\title{
Estimates of genetic parameters for traits measured in Phase A, C and D of the South African performance testing scheme.
}

\author{
M.J. Bradfield $\#$, B.E. Mostert, C.A.J. Exley, F. Jordaan and L. Bergh \\ ARC-Animal Improvement Institute, Private Bag X2,Irene, 0062, South Africa \\ "Email:michael@iapi.agric.za
}

\section{Introduction}

Numerous growth, reproduction, feed efficiency and shoulder measurement traits are recorded on beef cattle under the auspices of the South African Beef Cattle Performance Testing Scheme (NBCPTS). Best Linear Unbiased Prediction (BLUP) was first introduced to the South African beef industry on a national level in 1994, when univariate animal models were used to predict breeding values for traits measured in Phase A (birth and weaning weight) and B (yearling and 18 month weight) of the Scheme (Van der Westhuizen et al., 1995). In 1996 traits recorded in Phase D (growth tests on-farm) were also included into the analyses. Traits evaluated in Phase D include Average Daily Gain (ADGD), Kleiber Ratio (KR), Scrotum Circumference (SCD) and Shoulder Height (SHD). Although an univariate analysis uses all available pedigree information to calculate EBVs, it does not account for selection between traits, nor does it use the association (correlations) between traits to increase the accuracy of the EBVs. A common management practice in South Africa is to cull poor performing and functionally inefficient animals at weaning before further testing in Phase D, or alternatively, animals with above average performance are sent to central performance testing stations (Phase C). There is thus a high level of selection at this stage which should be accounted for in the national genetic evaluation. Traits measured in Phase $\mathrm{C}$ tests include Average Daily Gain (ADGC), Food Conversion Ratio (FCRC), Scrotum Circumference (SCC) and Shoulder Height (SHC). The objective of this study was to estimate covariance components between weaning weight and traits measured in Phase C and D of the NBCPTS. Results from this study will allow Phase C data to be included into the routine genetic evaluations, will account for the selection that occurs at weaning when analysing Phase C and D data, and will also increase the accuracy of EBVs by using the correlations between traits.

\section{Material and Methods}

Data from the Bonsmara and Angus breeds were used to estimate covariance components. The Bonsmara data set consisted of 31251 pedigree records whilst the Angus had 25501 pedigree records. Herds were required to be linked by sires that had at least 30 progeny in each of two or more herds and at least 200 Phase $\mathrm{C}$ and $\mathrm{D}$ records for the Bonsmara and 50 for the Angus. There were 21 Bonsmara and 12 Angus herds that met this criterion. Only data measured after 1985 for the Angus and 1990 for the Bonsmara was used in the analysis. Futher edits excluded single sire contemporary groups, embryo calves and twins. Traits considered in the analysis were: phase A: weaning weight additive (WWA), weaning weight maternal (WWM); phase C: ADGC, FCRC, SCC and SHC; phase D: ADGD, KR, SCD and SHD. Characteristics of the data structure are given in Table 1.

Fixed effects were modelled using ASREML (Gilmour, 1999) fitting the complete pedigree structure. Based on these results the contemporary group for weaning weight was defined as herd, year of birth, sex, weigh-date and user defined management group. Age at wean and age of dam were fitted as linear and quadratic covariables respectively. The contemporary group for traits measured in Phase $\mathrm{C}$ was defined as test center, test number, test year, test procedure and test phase. The contemporary group for traits measured in Phase D was defined as weaning weight contemporary group, test number, test year, date at start of test and test phase. Only sires were considered in the Phase $\mathrm{C}$ and $\mathrm{D}$ analysis. Age at start of test and age of dam were fitted as linear and quadratic covariables respectively, for both Phase $\mathrm{C}$ and $\mathrm{D}$. For random effects univariate analyses using ASREML (Gilmour, 1999) were carried out and Log Likelihood Ratio Tests were done in order to assess the importance of maternal and permanent environmental effects, and to obtain starting values to use in subsequent multivariate analyses. The multivariate analyses fitted the random effects of additive genetic, maternal genetic and permanent environment, for the trait weaning weight. For traits measured in Phase $\mathrm{C}$ and $\mathrm{D}$ only the additive genetic effect was fitted. The multivariate analyses was done using a combination of tri-variate and multivariate analyses (up to five traits simultaneously) to cover the full range of covariances. Overlapping of traits meant that some particular values were estimated two or three times and an average value for the trait was used in this instance. 
(C) South African Society of Animal Science

Short paper and poster abstracts: $38^{\text {th }}$ Congress of the South African Society of Animal Science

Table 1 Characteristics of the data structure

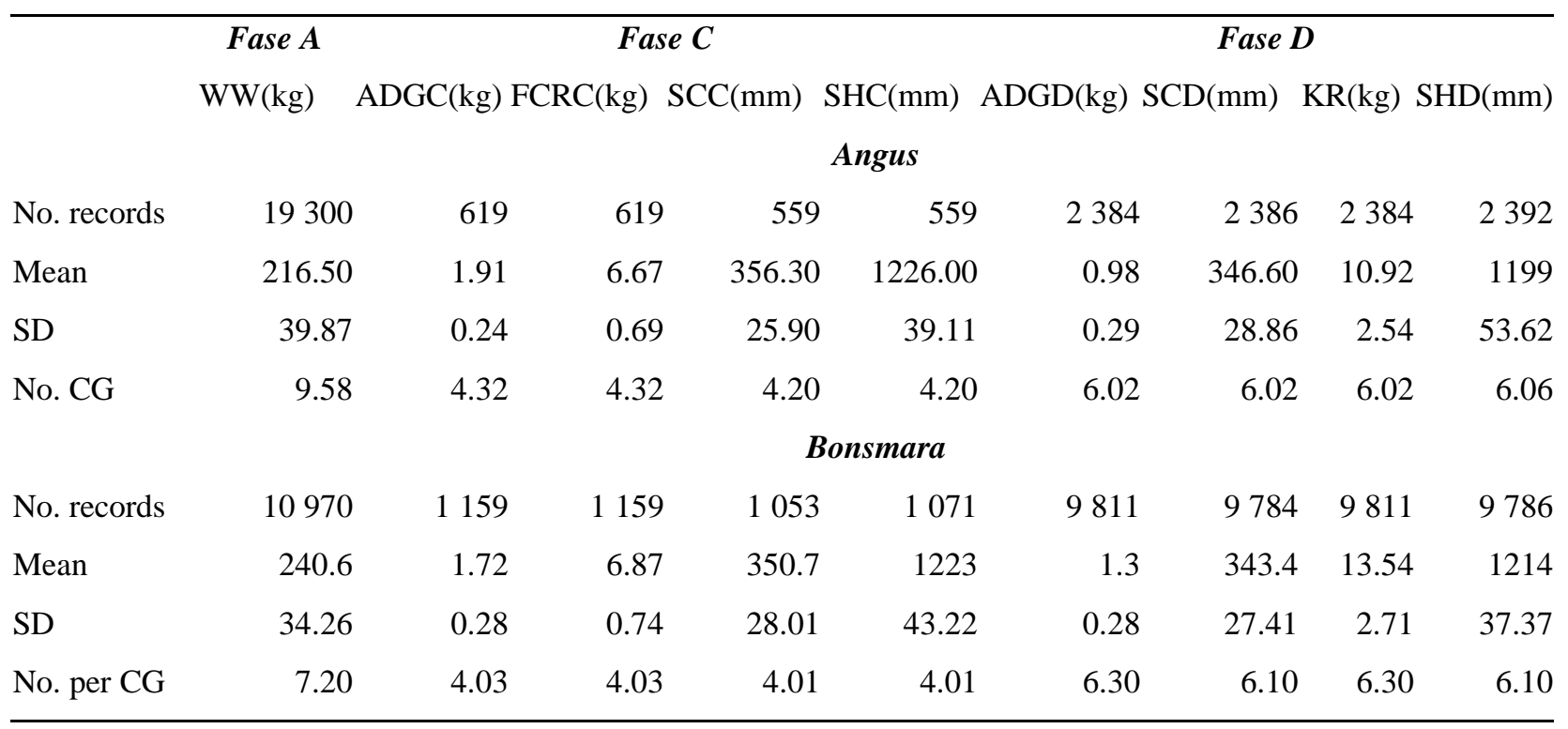

\section{Results and Discussion}

Heritability estimates for the Angus were generally higher than those observed for the Bonsmara breed (Table 2). Genetic correlations were generally similar in magnitude between both the Angus and Bonsmara breeds except for the correlations between SCC and FCRC where there was a positive correlation of 0.15 for the Angus and a slight negative correlation of -0.02 for the Bonsmara. Another exception was the correlation of -0.22 between ADGD and SCC in the Angus and 0.02 in the Bonsmara respectively. Scrotal Circumference and Shoulder Height had, as expected, very high correlations between Phase C and D (0.64 - 0.99) and Log-Likelihood Ratio Test showed that most of these correlations were not significantly different to unity. Correlation's were also highly positive in both breeds between WWA and ADGC and WWA and ADGD (0.63 and 0.75 respectively). Although negative, the correlations between WWA and FCRC of -0.36 and -0.25 are favourable. Environmental correlations were markedly similar across the two breeds within Phase C and D. Environmental correlations between traits measured in Phase $\mathrm{C}$ and $\mathrm{D}$ were zero as these traits were not measured on the same animal. Reasons for the low heritability estimate of 0.15 for WWA in the Bonsmara compared to 0.29 in the Angus requires further investigation. The estimate of 0.15 is much lower than the mean estimate of 0.35 taken from 172 studies reported in the review by Koots et al. (1994). The difference between the heritability estimates of ADGC and FCRC in the Angus compared to the Bonsmara, is difficult to explain and could possibly be attributed to environmental effects that are unaccounted for in the measurement of ADG. Despite the differences in estimates for these specific traits, heritability estimates for the other traits were generally within or close to the ranges of estimates reported in the review by Koots et al. (1994). For most traits the genetic correlations are favourable and suggest that selection for one trait will not adversely affect the other. Of note is the favourable negative correlation between WWA and FCRC. These parameter estimates will be refined as more Phase $\mathrm{C}$ data becomes available and the reason for the low heritability estimate for the Bonsmara becomes known. 
Table 2 Variance component estimates for the Angus and Bonsmara breeds respectively. Heritability estimates are given on the diagonal, genetic correlation's above and environmental correlations below.

\begin{tabular}{|c|c|c|c|c|c|c|c|c|c|}
\hline & \multicolumn{9}{|c|}{ Angus } \\
\hline & WWA & $A D G C$ & $F C R C$ & $S C C$ & $S H C$ & $A D G D$ & $S C D$ & $K R$ & SHD \\
\hline$W W A$ & 0.29 & 0.63 & -0.36 & 0.37 & 0.57 & 0.44 & 0.59 & -0.10 & 0.84 \\
\hline$A D G C$ & 0.23 & 0.47 & -0.63 & 0.19 & 0.36 & 0.43 & 0.40 & 0.11 & 0.46 \\
\hline FCRC & 0.03 & -0.75 & 0.21 & 0.15 & -0.02 & -0.08 & -0.10 & 0.18 & -0.25 \\
\hline$S C C$ & 0.21 & 0.21 & 0.02 & 0.70 & 0.13 & -0.22 & 0.99 & -0.05 & 0.00 \\
\hline$S H C$ & 0.33 & 0.63 & -0.44 & 0.11 & 0.61 & 0.51 & 0.03 & -0.04 & 0.88 \\
\hline$A D G D$ & 0.24 & 0 & 0 & 0 & 0 & 0.28 & 0.10 & 0.91 & 0.48 \\
\hline$S C D$ & 0.24 & 0 & 0 & 0 & 0 & 0.43 & 0.48 & -0.13 & 0.34 \\
\hline$K R$ & -0.08 & 0 & 0 & 0 & 0 & 0.92 & 0.29 & 0.26 & 0.78 \\
\hline$S H D$ & 0.41 & 0 & 0 & 0 & 0 & 0.35 & 0.18 & 0.24 & 0.56 \\
\hline
\end{tabular}

\begin{tabular}{|c|c|c|c|c|c|c|c|c|c|}
\hline & \multicolumn{9}{|c|}{ Bonsmara } \\
\hline & $W W A$ & $A D G C$ & $F C R C$ & $S C C$ & $S H C$ & $A D G D$ & $S C D$ & $K R$ & SHD \\
\hline$W W A$ & 0.15 & 0.75 & -0.25 & 0.06 & 0.73 & 0.42 & 0.32 & 0.05 & 0.77 \\
\hline$A D G C$ & 0.23 & 0.22 & -0.60 & 0.42 & 0.66 & 0.78 & 0.10 & 0.65 & 0.53 \\
\hline FCRC & -0.03 & -0.68 & 0.42 & -0.02 & -0.06 & -0.43 & 0.06 & 0.14 & -0.27 \\
\hline$S C C$ & 0.24 & 0.17 & -0.07 & 0.51 & 0.32 & 0.02 & 0.64 & 0.00 & 0.0004 \\
\hline$S H C$ & 0.37 & 0.25 & -0.04 & 0.05 & 0.46 & 0.40 & 0.27 & 0.27 & 0.99 \\
\hline$A D G D$ & 0.21 & 0 & 0 & 0 & 0 & 0.30 & 0.24 & 0.49 & 0.49 \\
\hline$S C D$ & 0.25 & 0 & 0 & 0 & 0 & 0.30 & 0.37 & 0.13 & 0.18 \\
\hline$K R$ & -0.10 & 0 & 0 & 0 & 0 & 0.82 & 0.21 & 0.28 & 0.27 \\
\hline$S H D$ & 0.37 & 0 & 0 & 0 & 0 & 0.35 & 0.17 & 0.20 & 0.39 \\
\hline
\end{tabular}

\section{Conclusions}

Results from this study will however allow Phase $\mathrm{C}$ data to be included into the routine national genetic evaluations, will account for the selection that occurs at weaning when analysing Phase $\mathrm{C}$ and $\mathrm{D}$ data, and will also increase the accuracy of EBVs by using correlations between traits.

\section{References}

Gilmour, A. R., 1999. ASREML user guide.

Koots, K.R. et al., 1994. ABA. 64, 309.

Van der Westhuizen, J. et al., 1995. Proc. $34^{\text {th }}$ Annual Congr. SASAS. Bloemfontein. pp 81. 